\title{
Light trapping of crystalline Si solar cells by use of nanocrystalline Si layer plus pyramidal texture
}

\author{
Kentaro Imamura, Nonaka Takaaki, Yuya Onitsuka, \\ Daichi Irishika, and Hikaru Kobayashi* \\ Institute of Scientific and Industrial Research, Osaka University, 8-1 \\ Mihogaoka, Ibaraki, Osaka 567-0047, Japan
}

The surface structure chemical transfer (SSCT) method has been applied to fabrication of single crystalline Si solar cells with $170 \mu \mathrm{m}$ thickness. The SSCT method, which simply involves immersion of $\mathrm{Si}$ wafers in $\mathrm{H}_{2} \mathrm{O}_{2}$ plus $\mathrm{HF}$ solutions and contact of Pt catalyst with Si taking only $\sim 30$ s for 6 inches wafers, can decrease the reflectivity to less than $3 \%$ by the formation of a nanocrystalline Si layer. However, the reflectivity of the nanocrystalline Si layer/flat Si surface/rear Ag electrode structure in the wavelength region longer than $1000 \mathrm{~nm}$ is high because of insufficient absorption of incident light. The reflectivity in the long wavelength region is greatly decreased by the formation of the nanocrystalline $\mathrm{Si}$ layer on pyramidal textured Si surfaces due to an increase in the optical path length. Deposition of phosphosilicate glass (PSG) on the nanocrystalline Si layer for formation of pn-junction does not change the ultralow reflectivity because the surface region of the nanocrystalline Si layer possesses a refractive index of 1.4 which is nearly the same as that of PSG of 1.4 1.5. The PSG layer is found to passivate the nanocrystalline Si layer, which is evident from an 
increase in the minority carrier lifetime from 12 to $44 \mu \mathrm{s}$. Hydrogen treatment at $450{ }^{\circ} \mathrm{C}$ further increases the minority carrier lifetime approximately to doubled value. The solar cells with the <front Ag electrode/nanocrystalline $\mathrm{Si}$ layer/pyramidal $\mathrm{Si}$ substrate/boron-diffused back surface field/Ag rear electrode> structure show a high conversion efficiency of $18.5 \%$ in spite of the simple cell structure without antireflection coating. In this case, the short circuit photocurrent density is $40.1 \mathrm{~mA} / \mathrm{cm}^{2}$ under AM1.5 $100 \mathrm{~mW} / \mathrm{cm}^{2}$ irradiation.

*Corresponding author. E-mail address: $\underline{\text { h.kobayashi@sanken.osaka-u.ac.jp }}$ 


\section{Introduction}

The reflectivity of flat $\mathrm{Si}$ surfaces is high in the range between $\sim 30 \%$ (at $800 \mathrm{~nm}$ wavelength light) and $\sim 50 \%$ (at $300 \mathrm{~nm}$ ) because of the large difference in the refractive indexes between air (i.e., 1) and Si (3.7 at $800 \mathrm{~nm}$ and 5.6 at $400 \mathrm{~nm}$ ). The conventional method to decrease the reflectivity of Si surfaces employs formation of pyramidal structure for single crystalline $\mathrm{Si}$ solar cells, which structure is formed by alkaline etching (e.g., $\mathrm{KOH}$ or $\mathrm{NaOH}$ plus alcohol such as isopropanol [1], butanol [2], etc.). Alkaline etching utilizes anisotropic etching, i.e., the etching rate of $\mathrm{Si}$ is much higher in the (100) direction than that in the (111) direction. In the case of poly-crystalline $\mathrm{Si}$ (poly-Si) solar cells, however, various surface orientations other than the (100) surface are present, and thus anisotropic alkaline etching cannot form uniform pyramidal structure. Therefore, for poly-Si solar cells, acid etching (i.e., $\mathrm{HNO}_{3}$ plus $\mathrm{HF}$ solutions) [3,4] is employed to produce textured surfaces. These textured surfaces utilize multiple reflection to decrease the reflectivity, but light reflected twice at Si surfaces and reflected light with low incident angle go out. Consequently, the reflectivity cannot be made very low, i.e., above $\sim 10 \%$ and $\sim 20 \%$ for single crystalline and poly-Si wafers, respectively. Another method to produce textured Si surfaces employs e.g., reactive ion etching [5], but the fabrication cost is much higher than those for wet-etching.

Insufficient light absorption is another problem arising from thin $\mathrm{Si}$ wafers for solar cell use (wafer thickness of commercial crystalline Si solar cells: 180 130 $\mathrm{\mu m}$ ). To avoid this problem, light should effectively be 
confined in Si. The conventional pyramidal structure possesses light trapping effect due to total reflection (i.e., light with incident angle larger than $\sim 16^{\circ}$ is totally reflected at $\mathrm{Si}$ surfaces without going out of $\mathrm{Si}$ ).

It has recently been shown that micro- plus nano-order structures on $\mathrm{Si}$ surfaces can achieve ultralow reflectivity. Nano-structures are produced on pyramidal textured Si surfaces by use of metal-catalyzed method [6,7], etching in $\mathrm{HNO}_{3}$ plus $\mathrm{HF}$ solutions [8], electrochemical etching [9], etc. Deposition of needle-like Er-doped $\mathrm{ZnO}$ structure on silicon nitride film-covered pyramidal textured Si substrates can also achieve ultralow reflectivity [10].

We have recently developed a fabrication method for ultralow reflectivity crystalline Si wafers which method simply includes contact of platinum (Pt) catalyst with $\mathrm{Si}$ wafers immersed in a hydrogen peroxide $\left(\mathrm{H}_{2} \mathrm{O}_{2}\right)$ plus hydrofluoric acid $(\mathrm{HF})$ solution called surface structure chemical transfer (SSCT) method [11-14]. In the present study, a nanocrystalline Si layer has been produced on a pyramidal Si surface by use of the SSCT method, and it is clearly shown that this structure possesses excellent light trapping effect. Using the nanocrystalline Si layer/pyramidal structure, high conversion efficiency of $18.5 \%$ and high short-circuit photocurrent density of $40.1 \mathrm{~mA} / \mathrm{cm}^{2}$ have been achieved in spite of simple solar cell structure without antireflection coating. The short-circuit photocurrent density of $40.1 \mathrm{~mA} / \mathrm{cm}^{2}$ is about $94 \%$ of the theoretical limit [15].

\section{Experiments}


Crystalline Si wafers used in this study were i) polished boron-doped p-type Si wafers and ii) pyramidal textured boron-doped p-type Si wafers. The polished Si wafers had a thickness of $725 \mu \mathrm{m}$ and a resistivity of $7 \sim 12$ Scm. The pyramidal textured surface structure was produced by use of anisotropic alkaline etching after removal of a damaged layer introduced during slicing. The thickness and the resistivity of the pyramidal textured Si wafers were $170 \mu \mathrm{m}$ and $3 \sim 4 \Omega \mathrm{cm}$, respectively. After the RCA cleaning, the $\mathrm{Si}$ wafers were immersed in $15 \mathrm{wt} \% \mathrm{H}_{2} \mathrm{O}_{2}$ plus $25 \mathrm{wt} \% \mathrm{HF}$ solutions at room temperature, and Pt catalyst installed on a roller was contacted with the Si wafers. The period of contact of the Si wafer with the Pt catalyst was between $10 \mathrm{~s}$ and $30 \mathrm{~s}$. After rinse with ultra-pure water, phosphosilicate glass (PSG) and borosilicate glass (BSG) were deposited on the front and rear Si surfaces, respectively, using a spin coating method, followed by heat treatment at $925{ }^{\circ} \mathrm{C}$ for $30 \mathrm{~min}$ in nitrogen. PSG and BSG were used for fabrication of pn-junction and back surface field (BSF), respectively. Front silver (Ag) electrodes were fabricated by use of an Ag paste, and rear Ag electrodes were formed using a vacuum deposition method after removal of the BSG layer. Then, the specimens were annealed in 5 vol\% hydrogen plus $95 \mathrm{vol} \%$ nitrogen atmosphere at $400{ }^{\circ} \mathrm{C}$ for $10 \mathrm{~min}$.

Reflectivity of Si surfaces was measured using a JASCO V-670 UV-visible spectrometer with an integrating sphere. Scanning electron microscopy (SEM) measurements were performed using a JEOL JSM-6335F field emission scanning electron microscope. Transmission electron microscopy (TEM) measurements were carried out with a JEOL EM-3000F 
transmission electron microscope with $300 \mathrm{keV}$ incident electron energy. Effective carrier lifetime was measured at an injection level of $1.5 \times 10^{14} \mathrm{~cm}^{-3}$ using a Sinton Instruments WCT-120 apparatus. Photocurrent density vs. photovoltage $\left(\mathrm{J}_{\mathrm{ph}}-\mathrm{V}_{\mathrm{ph}}\right)$ curves were observed under AM $1.5100 \mathrm{~mW} / \mathrm{cm}^{2}$ irradiation using a YAMASHITA DENSO YSS-50A solar simulator. Quantum efficiency was measured by use of a Bunkoukeiki BQE-100 spectrometer.

\section{Results and discussion}

Figure 1 shows the experimental set-up for production of ultralow reflectivity Si surfaces. Si wafer with a size of $5 \times 5 \mathrm{~cm}^{2}(\mathrm{~A})$ is placed in a hard vinyl chloride container (B) with an $\mathrm{H}_{2} \mathrm{O}_{2}$ plus $\mathrm{HF}$ mixed solution (C). A Pt catalyst (D) is attached to a roller (E) and by rotating the handle $(\mathrm{F})$, the Si wafer moves in the forward $(\mathrm{G})$ and reverse $(\mathrm{H})$ directions, contacting the Pt catalyst. By the contact of the Si wafer with the Pt catalyst, extremely fast surface reaction proceeds, and a 6 inches $\mathrm{Si}$ wafer can be treated within $30 \mathrm{~s}$ (cf. period of formation of pyramidal Si structure by alkaline etching: 20 30 $\min [1])$. Hereafter, this treatment is called the surface structure chemical transfer (SSCT) treatment.

Figure 2 shows the reflectivity of polished single crystalline Si wafers before and after the SSCT treatment. The Si wafers before the treatment had reflectivity between 30 and $65 \%$ (curve a), and it was greatly decreased by the SSCT treatment for $10 \mathrm{~s}$ (curve b). The reflectivity was further reduced with increases in the treatment period (curve $\mathrm{c}$ and $\mathrm{d}$ ). The 
reflectivity of the Si wafer after the SSCT treatment for $30 \mathrm{~s}$ (curve d) was in the range between $0.96 \%$ and $3.76 \%$ in the $300 \sim 800 \mathrm{~nm}$ wavelength region.

The ultra-low reflectivity results from the formation of a nanocrystalline Si layer [12]. Figures 3a, b, and c show the cross-sectional TEM micrographs for the nanocrystalline Si layer formed on the Si surfaces by the SSCT treatment for $10 \mathrm{~s}, 20 \mathrm{~s}$, and $30 \mathrm{~s}$, respectively. The thickness of the nanocrystalline Si layer increased with the treatment period. We have recently observed that the porosity in the nanocrystalline Si layer decreases with the depth [14]. This decrease in the porosity causes a continuous increase in the refractive index with the depth, which results in the refractive indexes of the nanocrystalline Si layer in the surface and interface regions close to those of air (i.e., unity) and $\mathrm{Si}$, respectively. In the case of the SSCT treatment for $30 \mathrm{~s}$, a thick nanocrystalline Si layer is formed and surface porosity becomes very high due to the long reaction period. Consequently, the refractive index in the surface region becomes sufficiently low to achieve the ultralow reflectivity. For the SSCT treatment shorter than $30 \mathrm{~s}$, on the other hand, the refractive index in the surface region is not sufficiently low due to the insufficiently low porosity, causing reflection at the air/nanocrystalline Si layer interface. Assuming that the all reflection occurs at the air/nanocrystalline Si layer interface and the extinction coefficient of the nanocrystalline Si layer is negligibly low, the average refractive index in the surface region of the nanocrystalline Si layer in the wavelength range between 300 and $800 \mathrm{~nm}$ is estimated to be 1.61 for $10 \mathrm{~s}$ treatment, 1.44 for $20 \mathrm{~s}$ treatment, and 1.31 for $30 \mathrm{~s}$ treatment, by use of 
the Fresnel equation.

Figure 4 shows the reflectivity of single crystalline Si wafers with the pyramidal textured surfaces (except for curve d) measured before and after the SSCT method. Before the SSCT method (curve a), the reflectivity was in the range between 10 and $40 \%$. The SSCT treatment greatly decreased the reflectivity in the wavelength region below $1000 \mathrm{~nm}$. However, the reflectivity in the wavelength region longer than $\sim 950 \mathrm{~nm}$ was high in the case of the saw-damage etched Si surfaces even with the SSCT treatment for $30 \mathrm{~s}$ (curve d). The specimens for the reflectivity measurements possessed $\mathrm{Si} / \mathrm{Ag}$ structure and reflection occurred at the rear Ag electrode (not at the front surface). For the flat surface structure, optical path is short, leading to insufficient absorption of incident light in the long wavelength region with the low absorption coefficient [16], thus causing the high reflectivity. For the pyramidal structure with the SSCT method, on the other hand, the reflectivity is low even in the long wavelength region $(\geq 950 \mathrm{~nm})$, indicating sufficient absorption of incident light.

When a PSG layer was deposited on the Si surfaces treated with the SSCT method for $20 \mathrm{~s}$ followed by the heat treatment at $925{ }^{\circ} \mathrm{C}$ (curve c), the reflectivity was nearly unchanged $(\leq 3 \%)$ from that before PSG deposition (curve b). The refractive index of PSG is reported to be 1.4 1.5 [17], which is nearly the same as that of the nanocrystalline Si surface produced by the SSCT treatment for 20 s. Therefore, deposition of PSG on the nanocrystalline Si layer does not change the refractive index, leading to the unchanged reflectivity of less than $3 \%$. 
Figure 5 shows the cross-sectional SEM micrographs of the pyramidal Si surface region after the SSCT method. A considerably thick (i.e., 250 $\mathrm{nm}$ ) nanocrystalline Si layer was formed in the top region of the pyramidal structure. In the bottom region, on the other hand, the nanocrystalline $\mathrm{Si}$ layer was much thinner.

Figure 6 shows the expected reflection mechanism of the pyramidal structure with a nanocrystalline Si layer. When light with a low incident angle is reflected at the top region, it goes out of the pyramidal structure. In the presence of a thick nanocrystalline Si layer in the top region, on the other hand, reflection is almost completely prevented. In the bottom region, on the other hand, reflection occurs to some extent because of the small thickness of the nanocrystalline Si layer. However, even if light is reflected in the bottom region, it enters $\mathrm{Si}$ again, leading to a low effective reflectivity.

Figure 7 shows the optical path in the pyramidal structure with and without a nanocrystalline Si layer. Total reflection occurs when the following equation is satisfied:

$$
\sin \theta \geq \frac{1}{n}
$$

where $\theta$ is the incident angle and $n$ is the refractive index of Si. Eq. (1) means that total reflection occurs when the incident angle is larger than $16^{\circ}$ (for wavelength of $800 \mathrm{~nm}$ with the refractive index of 3.7) and $10^{\circ}$ (for wavelength of $400 \mathrm{~nm}$ with the refractive index of 5.6).

With a nanocrystalline Si layer on the pyramidal Si structure, light entering from Si to a nanocrystalline Si layer travels straight at the interface 
because the refractive index of the nanocrystalline Si layer near the interface is nearly the same as that of $\mathrm{Si}$. Due to the graded structure of the nanocrystalline Si layer (i.e., porosity decreasing with the depth), the refractive index continuously increases with the depth. Consequently, light is gradually refracted in the nanocrystalline $\mathrm{Si}$ layer. The difference between the angle of incidence from Si to the nanocrystalline Si layer (cf. $\theta_{1}$ in Fig. 7) and that at the nanocrystalline Si surface $\left(\theta_{i}\right)$ is determined simply by the difference in the refractive indexes between Si and air, but doesn't depend on the refractive index of the nanocrystalline Si layer (see appendix). This consideration shows that the nanocrystalline Si/pyramidal Si structure possesses the same light trapping effect as the pyramidal Si structure [18].

Figure 8 shows the minority carrier lifetime of the pyramidal $\mathrm{Si}$ structure. The minority carrier lifetime just after formation of the pyramidal structure was only $0.76 \mu \mathrm{s}$. After the SSCT method to produce a nanocrystalline Si layer, the minority carrier lifetime only slightly decreased to $0.64 \mu \mathrm{s}$ although the surface area greatly increased by its formation. This is probably due to formation of $\mathrm{Si}-\mathrm{H}$ bonds in $\mathrm{HF}$ plus $\mathrm{H}_{2} \mathrm{O}_{2}$ solutions, resulting in a decrease in the density of Si dangling bonds [19]. The minority carrier lifetime after deposition of PSG was increased to $0.77 \mu \mathrm{s}$, and the subsequent heat treatment to produce pn-junction further increased it to $44 \mu \mathrm{s}$. It is very likely that PSG well contacts Si nanocrystals after the heat treatment which melts PSG, leading to formation of chemical bonds with Si dangling bonds. Removal of PSG by HF etching decreased the minority carrier lifetime to $12 \mu \mathrm{s}$. After hydrogen treatment at $450{ }^{\circ} \mathrm{C}$ on 
the PSG deposited specimens, the minority carrier lifetime further increased to $88 \mu \mathrm{s}$, which made it possible to achieve high conversion efficiency of the nanocrystalline $\mathrm{Si} / \mathrm{p}$-Si solar cells, as described below.

Figure 9 shows the I-V curves under AM $1.5100 \mathrm{~mW} / \mathrm{cm}^{2}$ irradiation for the active area of the $\mathrm{p}$-Si-based solar cells with the $<\mathrm{Ag} /$ nanocrystalline Si/pyramidal textured $\mathrm{p}$-Si substrate/B-diffused BSF/Ag> structure. In spite of the simple solar cell structure without antireflection coating, considerably high conversion efficiency of $18.5 \%$ was achieved after surface passivation using PSG and hydrogen treatment (curve a). It is noted that PSG deposition followed by the heat treatment used for passivation of the nanocrystalline Si layer is not an additional procedure because this method is used for the formation of pn-junction. The short-circuit photocurrent density $\left(\mathrm{J}_{\mathrm{SC}}\right)$ was $40.1 \mathrm{~mA} / \mathrm{cm}^{2}$, and this high $\mathrm{J}_{\mathrm{SC}}$ was due to 1) ultralow reflectivity resulting from the formation of the nanocrystalline Si layer, and 2) effective light trapping of the nanocrystalline Si/pyramidal Si structure. However, the open-circuit photovoltage $\left(\mathrm{V}_{\mathrm{OC}}\right)$ was relatively low, i.e., $600 \mathrm{mV}$. Implied $\mathrm{V}_{\mathrm{OC}}$ for the front and rear structures were measured to be 633 and $591 \mathrm{mV}$, respectively, indicating that the low $\mathrm{V}_{\text {OC }}$ was due to poor BSF. We observed that formation of BSF using $\mathrm{Al}$ diffusion increased the implied $\mathrm{V}_{\mathrm{OC}}$ to $630 \mathrm{mV}$ due to the gettering effect [20]. The relatively high implied Voc for the front structure is due to effective passivation of the Si nanocrystal surfaces by PSG and hydrogen treatment [21,22]. Without surface passivation (curve b), the cell performance was not high, i.e., Voc of $555 \mathrm{mV}$, $\mathrm{J}_{\mathrm{SC}}$ of $36.7 \mathrm{~mA} / \mathrm{cm}^{2}, \mathrm{FF}$ of 0.72 , and the conversion efficiency of $14.6 \%$. 
Figure 10 shows the internal quantum efficiency (IQE) vs. the wavelength of the solar cells with and without surface passivation. It is clearly shown that passivation using PSG and hydrogen treatment greatly improved IQE in the wavelength region shorter than $\sim 600 \mathrm{~nm}$. The absorption coefficient of short wavelength light is high, and thus it is absorbed near the surface. In the presence of surface defect states, electron-hole pairs generated by short wavelength light have a high probability to recombine there, resulting in low IQE. Surface defect states are eliminated by the passivation procedures, leading to prevention of surface recombination.

\section{Conclusion}

The nanocrystalline Si/pyramidal Si structure has been fabricated using the SSCT method. This structure can achieve a high conversion efficiency of $18.5 \%$ and a high $\mathrm{J}_{\mathrm{SC}}$ (i.e., $40.1 \mathrm{~mA} / \mathrm{cm}^{2}$ under $\mathrm{AM} 1.5$ $100 \mathrm{~mW} / \mathrm{cm}^{2}$ irradiation) in spite of the simple cell structure without antireflection coating, and the high solar cell performance is attributable to the following reasons:

1) The nanocrystalline Si layer in the surface region possesses a refractive index of $\sim 1.4$ and it continuously increases with the depth, resulting in low surface reflectivity less than $3 \%$.

2) PSG has a refractive index in the range $1.4 \sim 1.5$, and thus, after its deposition on the nanocrystalline Si layer, the ultralow reflectivity is maintained. 
3) The nanocrystalline Si/pyramidal $\mathrm{Si}$ substrate structure possesses the same light trapping effect as that of pyramidal Si structure, leading to effective absorption even for long wavelength light $(\geq 950 \mathrm{~nm})$.

4) Deposition of PSG followed by heat treatment greatly improves minority carrier lifetime because of melt of PSG and its contact with the nanocrystalline $\mathrm{Si}$ surfaces, resulting in elimination of surface defect states. Minority carrier lifetime is further improved by hydrogen treatment, and implied $\mathrm{V}_{\mathrm{OC}}$ increases to $633 \mathrm{mV}$.

Acknowledgement

This work was supported by CREST, Japan Science and Technology

Agency.

Appendix

The optical path in a nanocrystalline Si layer can easily be calculated assuming the layered structure as shown in Fig. 7. In each layer, the refractive index is assumed to be constant, and thus, light travels straight in each layer. For the layered structure, the following equation is satisfied:

$$
n_{\text {air }} \sin \theta_{\text {air }}=n_{1} \sin \theta_{1}=n_{2} \sin \theta_{2}=n_{i} \sin \theta_{i}=n_{S i} \sin \theta_{S i},
$$

where $n_{\text {air }}$ is the refractive index of air, $n_{i}$ is the refractive index of the $\mathrm{i}$-th nanocrystalline Si layer, $\theta_{a i r}$ and $\theta_{S i}$ are the incident angle from air and the refractive angle to $\mathrm{Si}$, respectively, and $\theta_{i}$ is the refractive and incident 
angles in the i-th nanocrystalline Si layer. From Eq. (2), it is easy seen that the refractive angle to $\mathrm{Si}, \theta_{S i}$, is determined only by the refractive indexes of air, $n_{\text {air }}$, and $\mathrm{Si}, n_{S i}$, but not on that of the nanocrystalline $\mathrm{Si}$ layer. 
[1] P. K. Singh, R. Kumar, M. Lal, S. N. Singh, B.K. Das, Sol. Energy Mater. Sol. Cells, 70 (2001) 103-113.

[2] K. P. Pola and Z. Irena, Microsyst. Technol. 19 (2013) 635-643.

[3] D. H. Macdonald, A. Cuevas, M. J. Kerr, C. Samundsett, D. Ruby, S. Winderbaum, and A. Leo, Sol. Energy 76 (2004) 277-283.

[4] Y.-T. Cheng, J.-J. Ho, S.-Y. Tsai, Z.-Z. Ye, W. Lee, D.-S. Hwang, S.-H. Chang, C.-C. Chang, and K. L. Wang, Sol. Energy 85 (2011) 87-94.

[5] Y. Inomata, K. Fukui, K. Shirasawa, Sol. Energy Mater. Sol. Cells 48 (1997) 237-242.

[6] D. Z. Dimitrov and C.-H. Du, Appl. Surf. Sci. 266 (2013) 1-4.

[7] Y. Xiu, S. Zhang, V. Yelundur, A. Rohatgi, D. W. Hess, and C. P. Wong, Langmuir 24 (2008) 10421-10426.

[8] Z. Swlatek, E. Beltowska, W. Mazlarz, and F. Krok, Mater. Sci. Eng. B 101 (2003) 291-296.

[9] B. S. Kim, D. H. Lee, S. H. Kim, G. H. An, K. J. Lee, N. V. Myung, and Y.-H. Choa, J. Am. Ceram. Soc. 92 (2009) 2415-2417.

[10] B. Liu, S. Qiu, R. Hu, Y. Liao, N. Chen, and G. Du, Appl. Surf. Sci. 259 (2012) 705-710.

[11] K. Imamura, F.C. Franco, Jr., T. Matsumoto, H. Kobayashi, Appl. Phys. Lett. 103 (2013) 013110-1-4.

[12] D. Irishika, K. Imamura, and H. Kobayashi, Sol. Energy Mater. Sol. Cells, 141 (2015) 1-6.

[13] K. Imamura, T. Nonaka, D. Irishika, and H. Kobayashi, ECS Solid State Lett., 4 (2015) Q63-Q65. 
[14] K. Imamura, D. Irishika, and H. Kobayashi, to be submitted.

[15] T. Tiedje, E. Yablonovitch, G. D. Cody, and B. G. Brooks, IEEE Trans. Electron Dev., ED-31 (1984) 711-716.

[16] M. A. Green and M. J. Keevers, Progress in Photovoltaics: Research and Applications, 3 (1995) 189-192.

[17] L. Zambov, I. Nedev, and Y. Yanakiev, Solid-State Electron. 34 (1991) 1231-1237.

[18] V. Magnin, J. Harari,M. Halbwax, S. Bastide, D. Cherfi, and J.-P. Vilcot, Sol. Energy 110 (2014) 378-385.

[19] F. F. Jr. Corpuz, T. Matsumoto, W.-B. Kim, H. Kobayashi, Solid-State Lett., 1 (2012) 89-91.

[20] O. Porre, M. Stemmer, and M. Pasquinelli, Mater. Sci. Eng. B24 (1994) 188-191.

[21] C. L. Mayfield and M. H. Huda, Comp. Theoret. Chem. 1019 (2013) $125-131$.

[22] S. Honda, T. Mates, M. Ledinsky, J. Oswald, A. Fejfar, J. Kočka, T. Yamazaki, Y. Uraoka, and T. Fuyuki, Thin Solid Films 487 (2005) 152-156. 
Figure captions

Fig. 1 Experimental set-up for fabrication of ultra-low reflectivity Si wafers.

Fig. 2 Reflectivity of the single crystalline Si wafers with a flat surface: (a) before the SSCT treatment, and after the SSCT treatment for the following periods: (b) $10 \mathrm{~s}$, (c) $20 \mathrm{~s}$, and (d) $30 \mathrm{~s}$.

Fig. 3 Cross-sectional TEM micrographs of the single crystalline $\mathrm{Si}$ wafers with a flat surface after the SSCT treatment for the following periods: (a) 10 s, (b) $20 \mathrm{~s},(\mathrm{c}) 30 \mathrm{~s}$.

Fig. 4 Reflectivity of the single crystalline $\mathrm{Si}$ wafers with the pyramidal textured surfaces: (a) before the SSCT treatment, (b) after the treatment for 20 s, (c) after PSG deposition on specimen (b). Reflectivity of single crystalline Si wafers with a flat surface after the SSCT treatment for $30 \mathrm{~s}$ is shown in curve (d) for reference.

Fig. 5 Cross-sectional SEM micrographs of the single crystalline Si wafers with the pyramidal textured structure observed after the SSCT method.

Fig. 6 Reflection mechanism for pyramidal textured Si surfaces: (a) without a nanocrystalline Si layer, (b) with a nanocrystalline Si layer. 
Fig. 7 Optical path in the pyramidal Si structure: (a) without a nanocrystalline Si layer, (b) with a nanocrystalline Si layer.

Fig. 8 Minority carrier lifetime of the $\mathrm{Si}$ wafers with the pyramidal structure: (a) as-formed pyramidal textured surface, (b) after the SSCT procedure, (c) after deposition of the PSG layer, (d) after heat treatment of specimen (c) at $950^{\circ} \mathrm{C}$, (e) after hydrogen treatment of specimen (d) at $450^{\circ} \mathrm{C}$, (f) after removal of PSG of specimen (d).

Fig. 9 I-V curves under AM $1.5100 \mathrm{~mW} / \mathrm{cm}^{2}$ irradiation for the solar cell with the <Ag/nanocrystalline $\mathrm{Si}$ layer/n+"-Si/p-Si substrate/B-diffused BSF/Ag> structure: (a) with surface passivation using PSG followed by heating at $925{ }^{\circ} \mathrm{C}$ and hydrogen treatment at $450^{\circ} \mathrm{C}$, (b) without surface passivation using PSG.

Fig. 10 Internal quantum efficiency for the solar cell with the $<$ Ag/nanocrystalline Si layer/n+-Si/p-Si substrate/B-diffused BSF/Ag> structure: (a) with surface passivation using PSG followed by heating at $950^{\circ} \mathrm{C}$ and hydrogen treatment at $450^{\circ} \mathrm{C}$, (b) without surface passivation using PSG. 


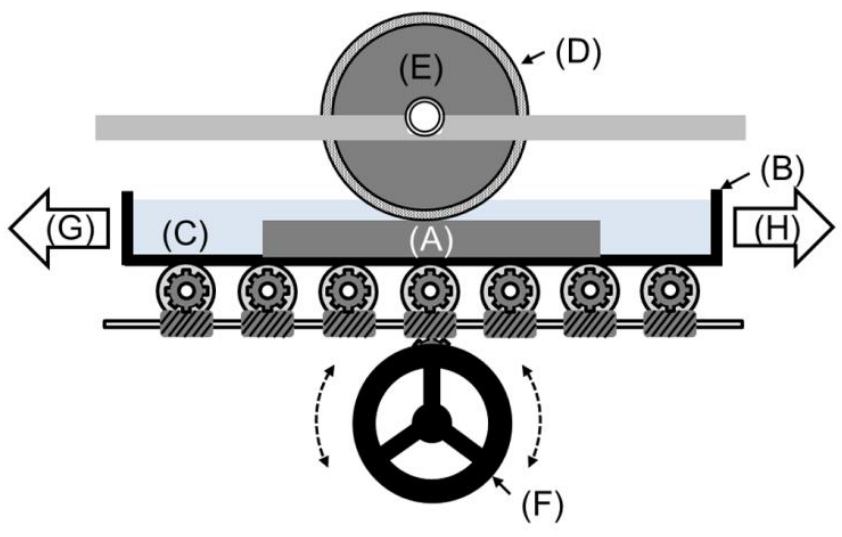

Fig.1

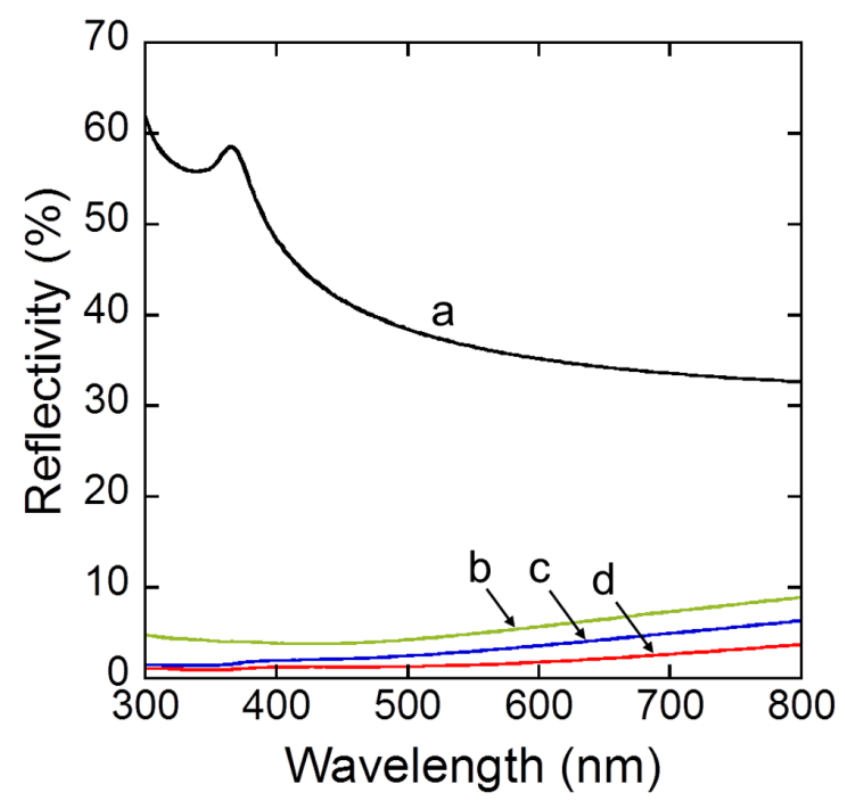

Fig.2

(a)

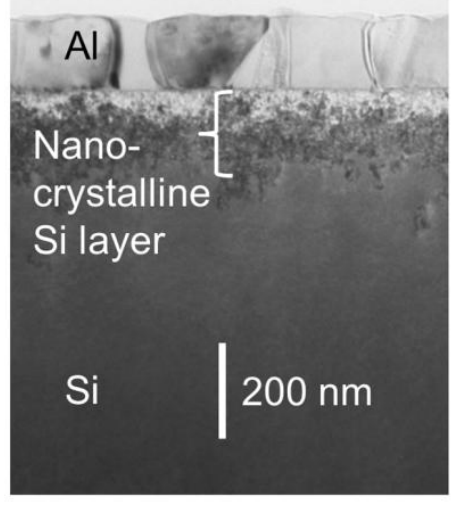

(b)

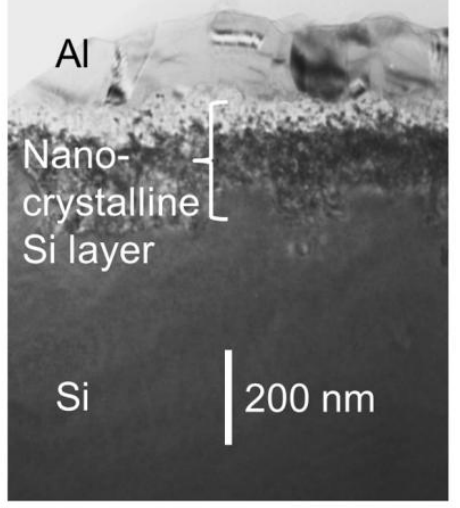

(c)

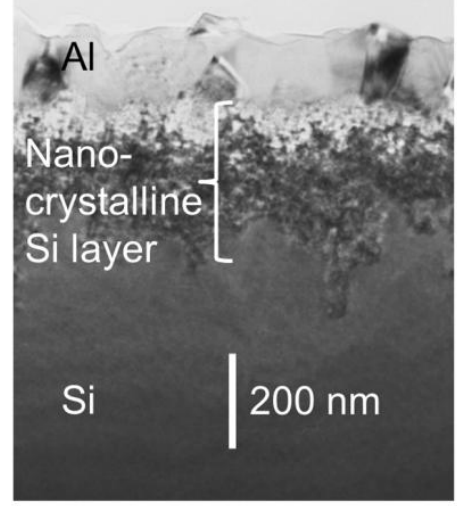

Fig.3 


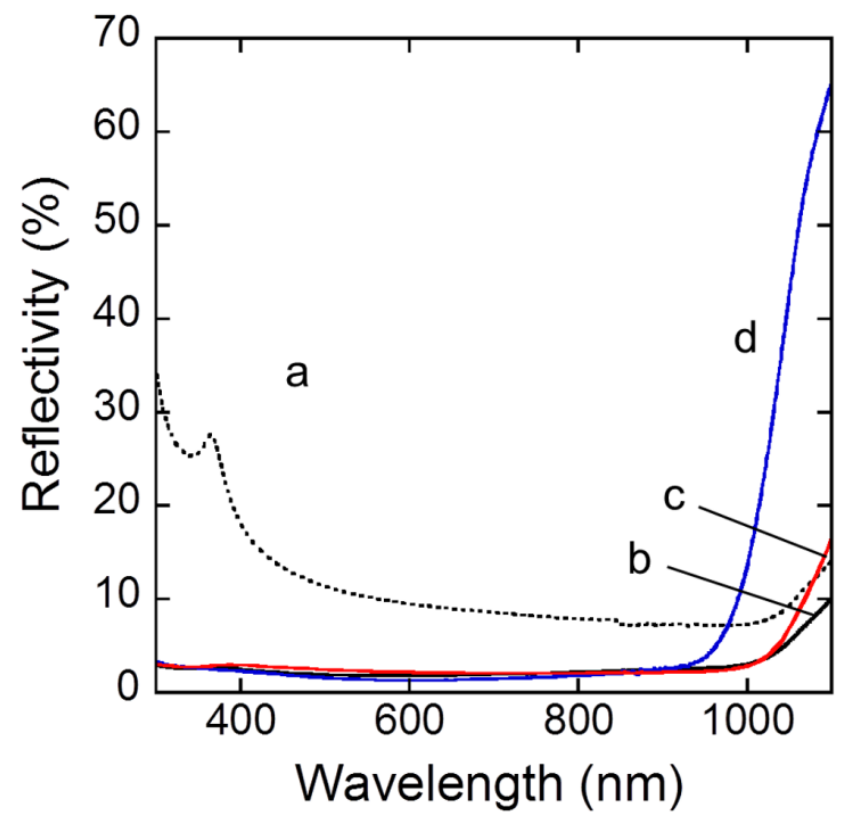

Fig.4

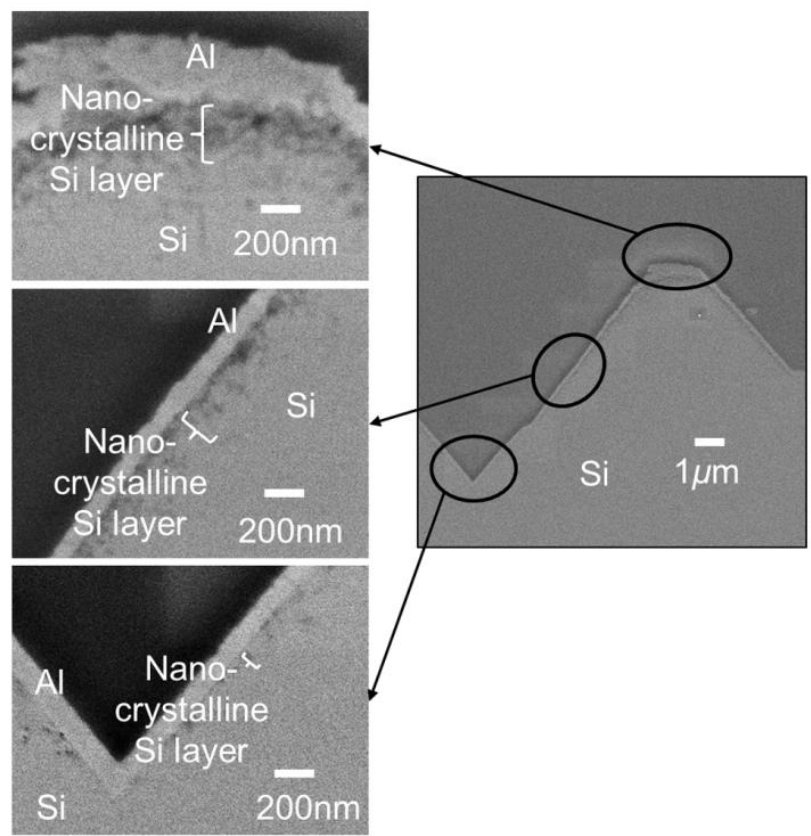

Fig.5 
(a) Incident light Reflected light

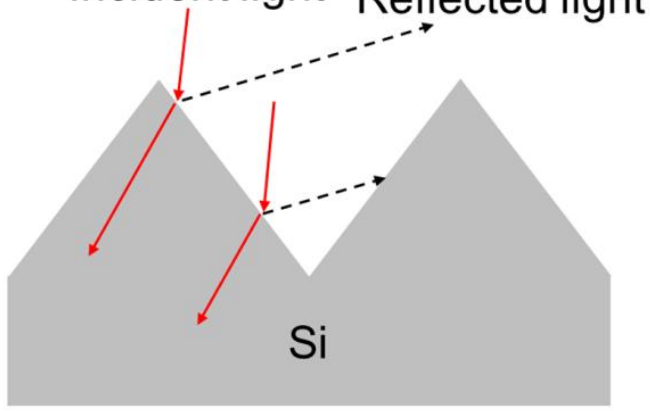

(b)

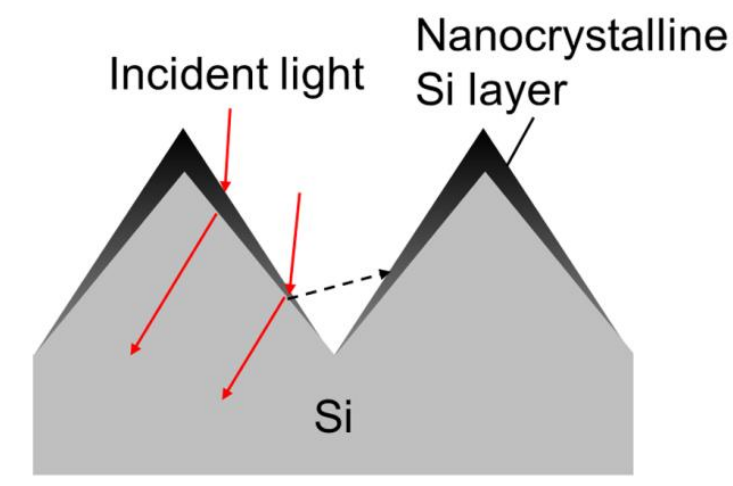

Fig.6

(a)

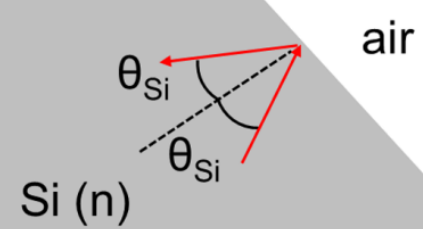

(b) Nanocrystalline Si layer

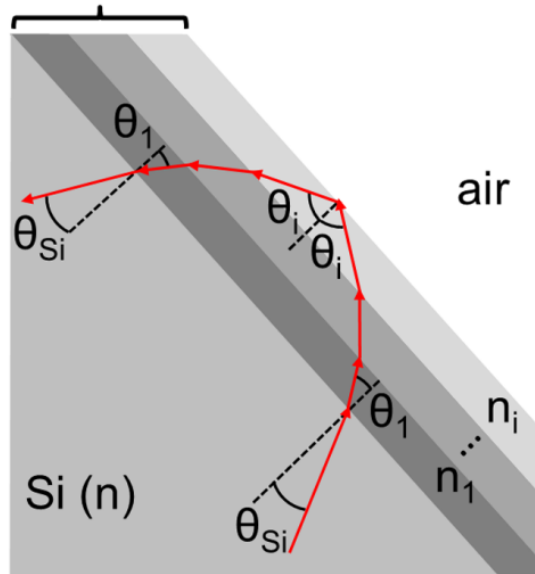

Fig.7 


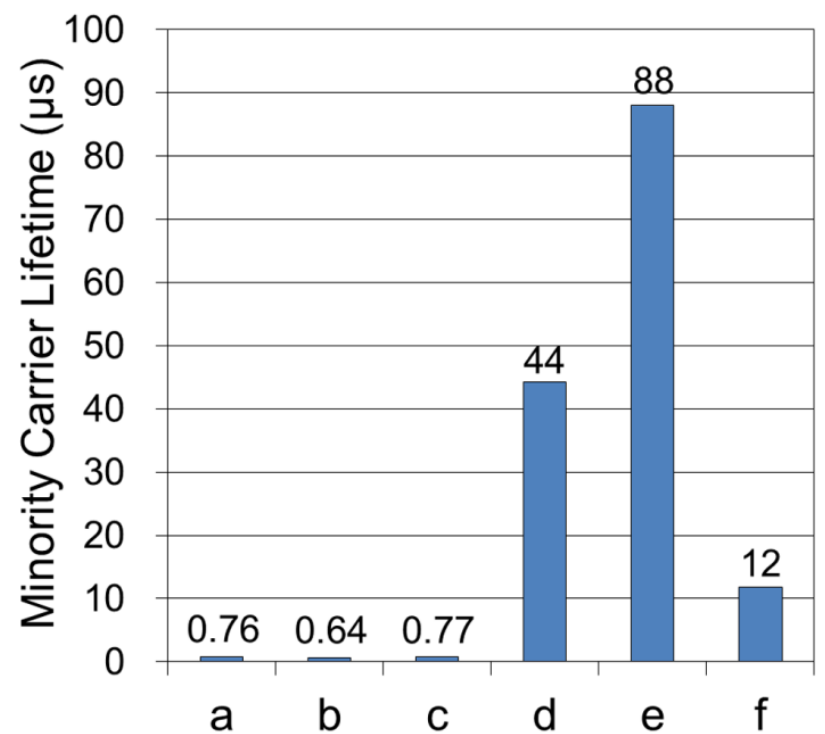

Fig.8

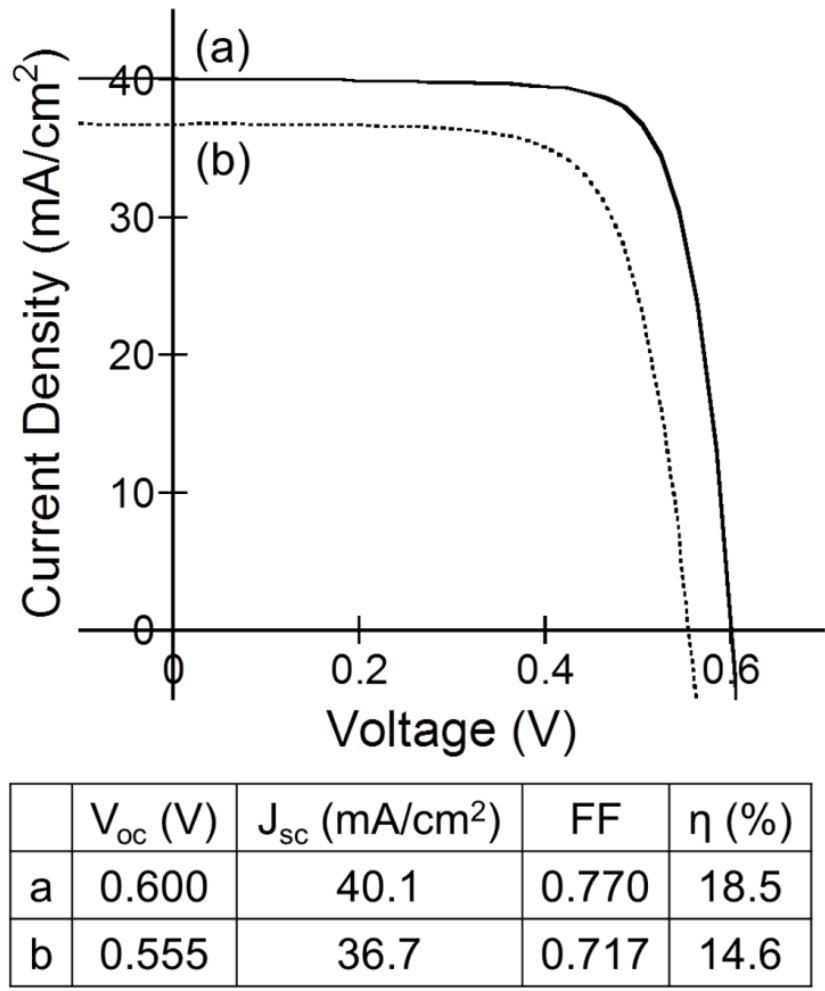

Fig.9 


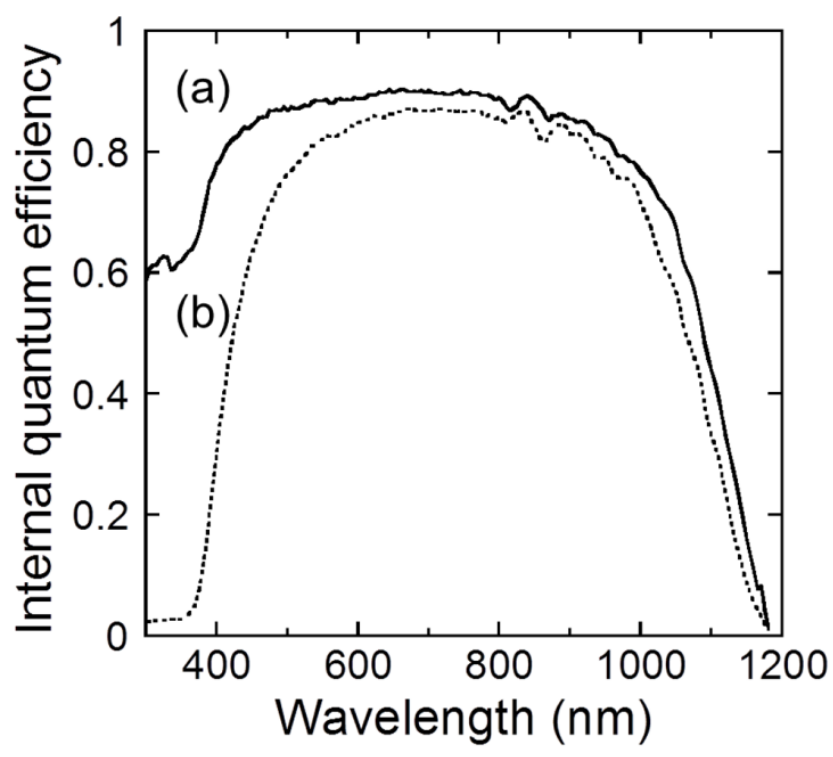

Fig.10 